\title{
Non-thermal emission from relativistic MHD simulations of pulsar wind nebulae: from synchrotron to inverse Compton ${ }^{\star}$
}

\author{
D. Volpi ${ }^{1}$, L. Del Zanna ${ }^{1}$, E. Amato ${ }^{2}$, and N. Bucciantini ${ }^{3}$
}

\author{
1 Dipartimento di Astronomia e Scienza dello Spazio, Università di Firenze, Largo E. Fermi 2, 50125 Firenze, Italy \\ e-mail: delia@arcetri.astro.it \\ 2 INAF - Osservatorio Astrofisico di Arcetri, Largo E. Fermi 5, 50125 Firenze, Italy \\ 3 Astronomy Department, University of California at Berkeley, 601 Campbell Hall, Berkeley, CA 94720-3411, USA
}

Received 18 January 2008 / Accepted 28 March 2008

\begin{abstract}
Aims. We develop a set of diagnostic tools for synchrotron-emitting sources, presented in a previous paper, to include a computation of inverse-Compton radiation from the same relativistic particles that give rise to the synchrotron emission. For the first time, we then study the gamma-ray emission properties of Pulsar Wind Nebulae, in the context of the axisymmetric jet-torus scenario.

Methods. We evolve the relativistic MHD equations and the maximum energy of the emitting particles, including adiabatic and synchrotron losses along streamlines. The particle energy distribution function is split into two components: one corresponds to radio-emitting electrons, which are interpreted to be a relic population that is born at the outburst of the supernova, and the other is associated with a wind population that is continuously accelerated at the termination shock and emits up to the gamma-ray band. The inverse Compton emissivity is calculated using the general Klein-Nishina differential cross-section and three different photon targets for the relativistic particles are considered: the nebular synchrotron photons, photons associated with the far-infrared thermal excess, and the cosmic microwave background.

Results. When the method is applied to the simulations that match the optical and X-ray morphology of the Crab Nebula, the overall synchrotron spectrum can only be fitted assuming an excess of injected particles and a steeper power law $\left(E^{-2.7}\right)$ with respect to previous models. The resulting $\mathrm{TeV}$ emission has then the correct shape but is in excess of the data. This is related to the magneticfield structure in the nebula, derived using simulations: in particular, the field is strongly compressed close to the termination shock, but with a lower than expected volume average. The jet-torus structure is also found to be visible clearly in high-resolution gamma-ray synthetic maps. We present a preliminary exploration of time variability in $\mathrm{X}$ - and gamma-rays. We find variations with timescales of about 2 years in both bands. The variability observed originates in the strongly time-dependent MHD motions inside the nebula.
\end{abstract}

Key words. radiation mechanisms: non-thermal - magnetohydrodynamics (MHD) - relativity - stars: pulsars: general ISM: supernova remnants - ISM: individual objects: Crab Nebula

\section{Introduction}

Pulsar wind nebulae (PWNe, or plerions) are a class of supernova remnants (SNR), which originates in the interaction between the ultra-relativistic wind blown by a pulsar (PW) and the surrounding supernova ejecta. The electromagnetic torques that act on a fast spinning, highly-magnetized neutron star, convert its rotational energy into the acceleration of a cold magnetized wind, which expands at relativistic velocity. The wind velocity needs to be reduced to match the boundary condition of nonrelativistic expansion of the confining supernova remnant. This occurs at a termination shock (TS), where the plasma is slowed down and heated, the magnetic field is amplified and the nebular particle spectrum is thought to be produced. If confinement is efficient, a non-negligible fraction of the energy lost by the pulsar, which is invisible as long as it is locked in the cold relativistic wind, may become detectable in the form of non-thermal radiation emitted by the relativistic particles in the nebula.

From the observational point of view, PWNe are characterized by the emission of a very broad band spectrum of nonthermal radiation, which typically extends from the radio to the

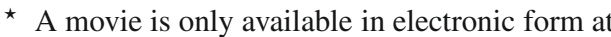
http://www . aanda. org
}

X-ray and even gamma-ray band. The primary emission mechanism is, at most frequencies, synchrotron radiation by relativistic particles gyrating in the nebular magnetic field. The synchrotron spectrum is cut off at some maximum frequency (typically that of soft gamma-rays) determined by the magnetic-field strength and the maximum energy that particles can attain inside the nebula. At higher photon energies, the dominant emission mechanism is, most likely, inverse Compton (IC) scattering by the same particles that interact with the different target photon fields. For reviews of theoretical as well as observational aspects of PWNe, see Gaensler \& Slane (2006), Kirk et al. (2007), and Bucciantini (2006).

The latest generation of Cherenkov detectors have shown PWNe to be among the highest energy emission sources in the Galaxy (e.g. Aharonian 2007; Gallant 2007). Detailed spatial mapping at X-ray frequencies, made possible using Chandra, showed that the peculiar axisymmetric morphology, known as jet-torus structure, initially seen in the prototype of the class, the Crab Nebula (Weisskopf et al. 2000; Hester et al. 2002), is also present in a number of other objects: the SNR associated to Vela (Helfand et al. 2001; Pavlov et al. 2003) and to PSR B1509-58 (Gaensler et al. 2002), G0.9+0.1 (Gaensler et al. 2001), G54.1+0.3 (Lu et al. 2002), and G130.7+3.1 (Slane et al. 2004). Relativistic motions and time-varying small-scale 
features (wisps around the TS and filaments in the torus) were also reported (Weisskopf et al. 2000; Hester et al. 2002; Pavlov et al. 2003). This paper intends to deal with all of these aspects, presenting for the first time a study of the highest energy emission from PWNe in the context of the jet-torus scenario, including an investigation of time variability.

The theoretical interpretation of the jet-torus morphology observed in the optical and X-ray bands is based on the idea that the pulsar wind energy flux is anisotropic, depending on latitude above the pulsar rotational equator (Bogovalov \& Khangoulian 2002; Lyubarsky 2002). An energy flux that reaches a maximum in the equatorial plane and decreases towards the polar axis causes the termination shock to be oblate, closer to the pulsar at the poles (Komissarov \& Lyubarsky 2003; Del Zanna et al. 2004). Understanding the formation of a torus in the equatorial plane and of jets along the polar axis is straightforward in this scenario, where the post-shock flows first converge toward the equator and are then diverted along the symmetry axis by magnetic hoop-stresses. Axisymmetric simulations in the relativistic MHD regime (RMHD) were able to fully confirm this view (Komissarov \& Lyubarsky 2004; Del Zanna et al. 2004).

The validity of this dynamical interpretation of the jet-torus structure was strengthened by the comparison between X-ray observations of the Crab Nebula and non-thermal emission maps built on top of the flow structure that results from RMHD simulations (Del Zanna et al. 2006). To complete this comparison, information on the particle spectrum as a function of position in the nebula is required. This type of information is unavailable in an MHD approach. Del Zanna et al. (2006) showed, however, that the evolution of the particle spectrum in the nebula, including synchrotron and adiabatic losses along streamlines, can be modeled, at least approximately, by a conservative MHD scheme, when an equation for the evolution of the maximum particle energy is developed inside the code. The general method proposed by Del Zanna et al. (2006) enables synchrotron radiation, polarization, and spectral-index maps, based on MHD simulations, to be computed. The resulting synchrotron surface-brightness maps, when compared with the data, showed that the axisymmetric MHD scenario is not only able to account for the general jet-torus morphology, but also to explain a number of finer scale details. Optical and X-ray spectral-index maps showed spectral hardening in the torus and softening toward the PWN border, which compare well with observations (Véron-cetty \& Woltjer 1993; Mori et al. 2004). Finally, the mildly-relativistic nebular flow, which contains velocities in the range of those observed along the jets and about the TS $(v \sim$ $0.5-0.8 \mathrm{c}$ ), produces features that closely resemble the rings and bright knot observed in the Crab Nebula, when Doppler boosting is properly taken into account.

The combination of RMHD simulations and diagnostic tools for synthetic synchrotron emission has therefore proven to be a powerful investigation technique. Unknown parameters such as the wind magnetization $\sigma$ (Kennel \& Coroniti 1984a), or the magnetic-field angular distribution, can be inferred by comparing theoretical predictions with observations. In Del Zanna et al. (2006), the Crab Nebula optical and X-ray morphology, in particular, were most appropriately described by a latitudeaveraged magnetization of $\sigma_{\text {eff }}=0.02$ and a narrow-striped wind region of low toroidal magnetic-field along the equator (runA). The energy-distribution function of relativistic electrons produced at the TS, was assumed to follow a single power law $E^{-(2 \alpha+1)}$ with $\alpha=0.6$, as in the model by Kennel \& Coroniti (1984b). No attempt to model radio emission was made. The resultant synchrotron spectrum showed an excess of flux in the
X-ray band (by a factor of two), and an unexpected flattening beyond $\approx 3 \times 10^{16} \mathrm{~Hz}$.

We investigate further why it is difficult to reproduce the observed high-energy synchrotron spectrum. We consider in particular whether this difficulty could be resolved by choosing a different particle distribution function. In contrast to the magnetic-field strength, the particle distribution function does not affect the dynamics, provided that the spectral index is higher than 0.5 , and can be changed without affecting the nebular morphology. While the synchrotron emission depends only on the magnetic field and particle distribution, the IC emission enables the relative contributions of the two, to be distinguished. To exploit this fact in the present paper, we extend the set of diagnostic tools for non-thermal radiation to the IC scattering process; we apply the method to our simulations (runA) that provide the most appropriate description of data for the Crab Nebula, extending the spectral coverage to the gamma-rays.

As we mentioned, an important development in the study of PWNe has been the detection of very high-energy gammaray emission from these objects. TeV emission has been detected from the Crab Nebula (Aharonian et al. 2004, 2006d; Albert et al. 2008), MSH 15-52 (Aharonian et al. 2005b), Vela (Aharonian et al. 2006a; Enomoto et al. 2006), Kookaburra complex (Aharonian et al. 2006b), HESS J1825-137 (Aharonian et al. 2006c), the composite SNR G 0.9+0.1 (Aharonian et al. 2005a), the two candidates HESS J1357-645, and HESS J1809193 (Aharonian et al. 2007). In this context, TeV emission can be generated, in principle, by two different processes: either inverse Compton emission, which involves relativistic electrons that up-scatter lower energy photons; or $\pi^{0}$ decay, which involves the presence of relativistic protons that produce pions by nuclear collisions. The presence of relativistic protons in PWNe is also supported by theoretical arguments. Particle acceleration at transverse relativistic shock waves, such as the pulsar wind TS, is difficult to reproduce using standard acceleration processes. The most successful model, so far, is based on resonant absorption by electrons and positrons, of the relativistic cyclotron-radiation produced by ions, which are predicted to be present in the pulsar wind. In the model, the ions are energetically-dominant in the wind, even though they are few in number.

The most likely mechanism to generate this emission is inverse Compton scattering of the same particles that produce the nebular synchrotron spectrum. These particles can up-scatter different targets: CMB photons, synchrotron-emitted photons, starlight, and possibly FIR photons due to reprocessing of the nebular radiation by dust within the SNR. Studying IC emission is important for two reasons. Firstly, modeling synchrotron and IC radiation at the same time, as expected, enables constraint of properties of the magnetic-field strength and particle number density, which cannot achieved using only synchrotron-emission data (Gould 1965; De Jager et al. 1996). A detailed modeling of the IC component is, at present, the only way to investigate whether the high-energy data can accommodate the requirement of a contribution of hadronic origin (e.g. Amato et al. 2003; Bednarek \& Bartosik 2003). The clarification of this point is important in view of the quest for galactic sources of cosmic rays at energies about the knee.

This paper presents a general method that could be applied to the entire class of PWNe and more generally to all non-thermal sources. It consists of the evolution in time and space, of the maximum energy of emitting particles, with other MHD dynamical variables, while the appropriate simulation set-up (e.g. initial and boundary conditions, resolution) and shape of the 
distribution function at injection sites, are arbitrary, and both can be chosen to be the most appropriate for each object under investigation. Our purpose is not to optimize the dynamical free parameters to reproduce specific nebulae, but to apply our emission model to the runA simulation and compare the results to the Crab Nebula data. The Crab Nebula is, in fact, the brightest PWN at very high energies, and is regarded as a standard candle for gamma-ray observations. This makes it a natural target for new instruments and a wealth of data is already available from ground and space instruments: EGRET (Nolan et al. 1993), COMPTEL (Kuiper et al. 2001), HEGRA (Aharonian et al. 2004), HESS (Masterson et al. 2005; Aharonian et al. 2006d), MAGIC (Albert et al. 2008). This year GLAST will be launched and so new data will be available from $20 \mathrm{MeV}$ to $300 \mathrm{GeV}$.

Existing gamma-ray observations are used to constrain the parameters of our model. The synthetic emission up to TeV energies is calculated for the first time based on time-dependent 2D numerical simulations. We assume in particular the configuration corresponding to runA, as reported in Del Zanna et al. (2006). Following Atoyan \& Aharonian (1996), we consider a more general distribution function of emitting electrons, which consists of two families: one corresponding to radio-emitting electrons, which can be interpreted as primordial population of particles, and the other corresponding to particles that are continuously accelerated at the termination shock, which is responsible for the synchrotron spectrum up to the gamma band. The IC emissivity is calculated in the optically thin case, which is appropriate for these objects, using the general Klein-Nishina differential cross-section. The three different photon targets, which are recognized as the most important for the Crab Nebula (Aharonian et al. 2006d), are considered: the nebular synchrotron photons, the photons responsible for the far-infrared thermal excess (Marsden et al. 1984), and the cosmic microwave background. Previous work applied similar emission recipes to stationary and radially-symmetric models (e.g. Gould 1979), and notably to the Kennel \& Coroniti (KC: Kennel \& Coroniti 1984a) RMHD model (De Jager \& Harding 1992; Atoyan \& Aharonian 1996).

The paper is structured as follows. In Sect. 2, our nonthermal emission model is presented. The integrated spectra, which are produced by both the KC model (used here to test the emission recipes) and our simulations, are shown in Sect. 3, and compared with observations of the Crab Nebula. In Sect. 4, we produce surface-brightness maps at different energies in the gamma-rays to compare with existing and future images. Finally, we study time variability in Sect. 5, using synthetic X-ray and gamma-ray emission.

\section{The non-thermal emission model}

We describe in detail the formulae used to compute synchrotron and IC emission. As mentioned in Sect. 1, the evolution of the distribution function and the synchrotron-emission recipes are adapted from Del Zanna et al. (2006), while the choice of the distribution function at the TS follows Atoyan \& Aharonian (1996). Approximations are necessary to avoid using formulae in which some terms are unknown. For instance, it is not possible, within the present scheme, to associate the local pressure value with the corresponding one (along streamlines) at the termination shock. This would require evolving the entire distribution function in both space and time, which is a task that is beyond the goal of this paper. As shown, a few additional approximations are used to reduce computational time, after checking that they do not change significantly the final results.

\subsection{Relativistic electrons}

In the Crab Nebula spectrum, two main breaks appear: one in the IR, and the other in the UV. To be able to account for both breaks, we consider the presence of two distinct populations of relativistic particles (electrons and positrons). In analogy with previous MHD models, we assume that the break in the UV is due to synchrotron cooling of a population, continuously accelerated at the TS, and responsible for the optical and higher frequency radiation (Kennel \& Coroniti 1984b). We then describe the radioemitting electrons as a different population, which has sometimes been interpreted as primordial (e.g. Atoyan \& Aharonian 1996), i.e. born at the outburst of the supernova. The distribution function of both populations is shaped following Atoyan \& Aharonian (1996).

Assuming isotropy, the radio-emitting particle distribution function per unit solid angle, is taken to be

$f_{\mathrm{r}}(\epsilon)=\frac{A_{\mathrm{r}}}{4 \pi} \epsilon^{-\left(2 \alpha_{\mathrm{r}}+1\right)} \exp \left(-\epsilon / \epsilon_{\mathrm{r}}^{*}\right)$,

which is assumed to be homogeneous in the PWN, and constant in time. Here, $\epsilon$ is the normalized energy of the particle (its Lorentz factor), $A_{\mathrm{r}}$ is the normalization constant, $\alpha_{\mathrm{r}}$ is the radio spectral index, and $\epsilon_{\mathrm{r}}^{*}$ is the radio cut-off energy, which is the energy corresponding to a synchrotron-cooling time comparable to the age of the nebula.

The second population is composed of the relativistic wind particles that are continuously accelerated at the termination shock and injected downstream into the nebula, with the following distribution function

$f_{0}\left(\epsilon_{0}\right)=\frac{A_{0}}{4 \pi}\left(\bar{\epsilon}+\epsilon_{0}\right)^{-\left(2 \alpha_{\mathrm{w}}+1\right)} \exp \left(-\epsilon_{0} / \epsilon_{\mathrm{w}}^{*}\right)$.

This must be evolved along streamlines in the PWN, and may change with time. The variables with the index 0 , are calculated at the TS. As for the previous case, $A_{0}$ is a constant to be determined based on the local number density or pressure, $\alpha_{\mathrm{w}}$ is the wind spectral index, and $\epsilon_{\mathrm{w}}^{*}$ is the cut-off energy, which corresponds to a maximum synchrotron frequency in the gamma-rays. The additional constant $\bar{\epsilon}$ represents the minimum energy of the wind population, which is chosen to match the infrared-optical synchrotron emission of the two families of electrons.

Following Del Zanna et al. (2006), adiabatic and synchrotron losses are taken into account in the evolution of the wind population, by defining the evolved distribution as

$f_{\mathrm{w}}(\epsilon)=\left(\frac{\rho}{\rho_{0}}\right)^{4 / 3}\left(\frac{\epsilon_{0}}{\epsilon}\right)^{2} f_{0}\left(\epsilon_{0}\right)$,

where $\rho$ is the rest mass density, and thus:

$f_{\mathrm{w}}(\epsilon)=\frac{A_{\mathrm{w}}}{4 \pi} p\left(\frac{\epsilon_{0}}{\epsilon}\right)^{2}\left(\bar{\epsilon}+\epsilon_{0}\right)^{-\left(2 \alpha_{\mathrm{w}}+1\right)} \exp \left(-\epsilon_{0} / \epsilon_{\mathrm{w}}^{*}\right)$.

We used the conservation of particle number along streamlines, and assumed adiabaticity for the pressure $\left(p \propto \rho^{4 / 3}\right)$. The wind normalization constant $A_{0}$ is equal to $A_{\mathrm{w}} p_{0}$, and we take the synchrotron losses into account using the quantity $\epsilon_{\infty}$, which is the maximum possible particle energy at a position along the streamline:

$\epsilon_{0}=\frac{\epsilon}{1-\epsilon / \epsilon_{\infty}}$.

The variables $\rho, p$ and $\epsilon_{\infty}$ are, in general, functions of space and time, and provided by our code. We note that adiabatic losses are taken into account only using the pressure term in Eq. (4), while we are forced to neglect them elsewhere (terms $\left(p / p_{0}\right)^{1 / 4}$ are approximated to 1), as explained in Del Zanna et al. (2006). 


\subsection{Synchrotron emission}

The synchrotron spectral power emitted by a single ultrarelativistic particle is

$\mathcal{P}_{v}^{\mathrm{SYN}}(v, \epsilon)=2 \sigma_{\mathrm{T}} c \frac{B_{\perp}^{2}}{8 \pi} \epsilon^{2} \delta\left(v-v_{\mathrm{m}}\right)$,

where $\sigma_{\mathrm{T}}$ is the Thomson cross-section, $B_{\perp}$ is the local magnetic field component normal to the particle's velocity, and $v$ is the observed frequency. We note that for simplicity we considered the monochromatic approximation, where $v_{\mathrm{m}}=0.29 v_{\mathrm{c}}$ is the location of the maximum emission (see e.g. Rybicki \& Lightman 1979), and the critical frequency is given by

$v_{\mathrm{c}}=\frac{3 e}{4 \pi m c} B_{\perp} \epsilon^{2}$

The emission coefficient is, in the general case

$j_{v}^{\mathrm{SYN}}(v)=\int \mathcal{P}_{v}^{\mathrm{SYN}}(v, \epsilon) f(\epsilon) \mathrm{d} \epsilon$,

whatever the distribution function $f(\epsilon)$. In accordance with the monochromatic approximation, we perform readily the above integration, and find

$j_{v}^{\mathrm{SYN}}(v)=2 \sigma_{\mathrm{T}} c \frac{[\epsilon(v)]^{3}}{2 v} \frac{B_{\perp}^{2}}{8 \pi} f[\epsilon(v)]$,

where

$\epsilon(v)=\left(0.29 \frac{3 e}{4 \pi m c} B_{\perp}\right)^{-1 / 2} v^{1 / 2}$.

It is easy to verify that, in the power-law regions of both the distribution functions described in the previous section, the emission coefficient behaves as $j_{v} \propto v^{-\alpha}$, as expected.

Due to relativistic beaming, the magnetic-field component in the above expressions, can be written as $B_{\perp}=|\boldsymbol{n} \times \boldsymbol{B}|$, where $\boldsymbol{n}$ is the line-of-sight direction. To reduce computational costs, we consider an isotropically-distributed field (discrepancies in the integrated fluxes are less than $3 \%$ at $1 \mathrm{keV}$ ), for which $B_{\perp}=$ $\sqrt{2 / 3} B / \gamma$, where $B$ is the toroidal magnetic field as measured in the laboratory frame, and $\gamma$ is the Lorentz factor of the fluid element. The synchrotron spectrum, which is expressed in terms of the total luminosity per unit frequency, is

$L_{v}^{\mathrm{SYN}}(v)=\int 4 \pi j_{v}^{\mathrm{SYN}}(\boldsymbol{r}, v) \mathrm{d} V$

where the integral is over the nebular volume.

Synthetic surface-brightness maps are derived by integrating the emissivity along the line of sight alone. The Doppler boosting towards the observer is taken in account in a similar way to the paper by Del Zanna et al. (2006).

\subsection{Inverse Compton emission}

The Inverse Compton spectral power emitted by a single ultrarelativistic particle with normalized energy $\epsilon$, may be written as

$\mathcal{P}_{v}^{\mathrm{IC}}(v, \epsilon)=c h v \int\left(\frac{\mathrm{d} \sigma}{\mathrm{d} v_{\mathrm{t}}}\right)_{\mathrm{IC}}\left(v_{\mathrm{t}}, v, \epsilon\right) n_{v}\left(v_{\mathrm{t}}\right) \mathrm{d} v_{\mathrm{t}}$,

where $n_{v}\left(v_{\mathrm{t}}\right)$ is the number density of target photons per unit frequency $v_{\mathrm{t}}$. The general form of the differential cross-section for IC scattering per unit frequency, which includes both the
Thomson and Klein-Nishina regimes, is given by Jones (1968); Blumenthal \& Gould (1970):

$\left(\frac{\mathrm{d} \sigma}{\mathrm{d} v_{\mathrm{t}}}\right)_{\mathrm{IC}}=\frac{3}{4} \frac{\sigma_{\mathrm{T}}}{\epsilon^{2} v_{\mathrm{t}}}\left[2 q \ln q+(1-q)\left(1+2 q+\frac{1}{2} \frac{(\Gamma q)^{2}}{1+\Gamma q}\right)\right]$,

with

$q=\frac{h v / m c^{2}}{\Gamma\left(\epsilon-h v / m c^{2}\right)}$ and $\Gamma=4 \epsilon h v_{\mathrm{t}} / m c^{2}$

Due to the kinematics of the scattering, $q$ is a parameter in the range $0<q \leq 1$ and $\Gamma$ determines the energy domain (the Thomson limit corresponds to $\Gamma \ll 1$ ).

As in the previous case, the emission coefficient is given by

$j_{v}^{\mathrm{IC}}(v)=\int \mathcal{P}_{v}^{\mathrm{IC}}(v, \epsilon) f(\epsilon) \mathrm{d} \epsilon$

for any distribution function of scattering particles $f(\epsilon)$. In the most general IC scattering case, we cannot reduce the above formula to an analytical expression and a double integral has to be computed numerically. Following Blumenthal \& Gould (1970), we replace the integration over $\epsilon$ by an integration over $q$. The relation between these two variables is

$\epsilon(q)=\frac{1}{2} \frac{h v}{m c^{2}}\left[1+\left(\frac{1+s q}{s q}\right)^{1 / 2}\right], \quad s=\frac{h v}{m c^{2}} \frac{h v_{\mathrm{t}}}{m c^{2}}$,

where the dimensionless quantity $s$ is small in the Thomson limit and large in the extreme Klein-Nishina regime.

The target photon density in Eq. (12) can have different origins. We study three cases, which are recognized to be relevant for the Crab Nebula and PWNe, in general (Atoyan \& Aharonian 1996): IC scattering of reprocessed synchrotron photons (IC-SYN), IC scattering of the far-infrared thermal radiation by local dust (IC-FIR), and IC scattering of cosmic microwave background photons (IC-CMB).

In the self-synchrotron emission case, the photon density at any position $\boldsymbol{r}$ can be determined using the radiative-transfer equation in the optically-thin regime (Gould 1979; De Jager \& Harding 1992; Atoyan \& Aharonian 1996):

$n_{v}^{\mathrm{IC}-\mathrm{SYN}}\left(\boldsymbol{r}, v_{\mathrm{t}}\right)=\frac{1}{c h v_{\mathrm{t}}} \int \frac{j_{v}^{\mathrm{SYN}}\left(\boldsymbol{r}^{\prime}, v_{\mathrm{t}}\right)}{\left|\boldsymbol{r}^{\prime}-\boldsymbol{r}\right|^{2}} \mathrm{~d} V^{\prime}$

where the spatial integral is over the entire nebula. To reduce computational costs, we use the approximation of spherical symmetry and a homogeneous synchrotron-emission coefficient, after testing that differences are negligible. We thus replace the above formula with

$n_{v}^{\mathrm{IC}-\mathrm{SYN}}\left(r, v_{\mathrm{t}}\right)=\frac{1}{c h v_{\mathrm{t}}} \frac{L_{v}^{\mathrm{SYN}}\left(v_{\mathrm{t}}\right)}{4 \pi R^{2}} U(r / R)$,

where the synchrotron spectral luminosity is given in Eq. (11), $R$ is the radius of the nebula, and

$U(x)=\frac{3}{2} \int_{0}^{1} \frac{y}{x} \ln \frac{x+y}{|x-y|} \mathrm{d} y$,

with $x=r / R$ and $y=r^{\prime} / R$. $U(x)$ is a non-dimensional quantity, decreasing with $x$ from 3 to 1.5 (Atoyan \& Aharonian 1996).

When the emission recipes are applied to the results of our simulations, the synchrotron photon density in Eq. (18) becomes a function of time. Variations in the synchrotron-emitted flux 

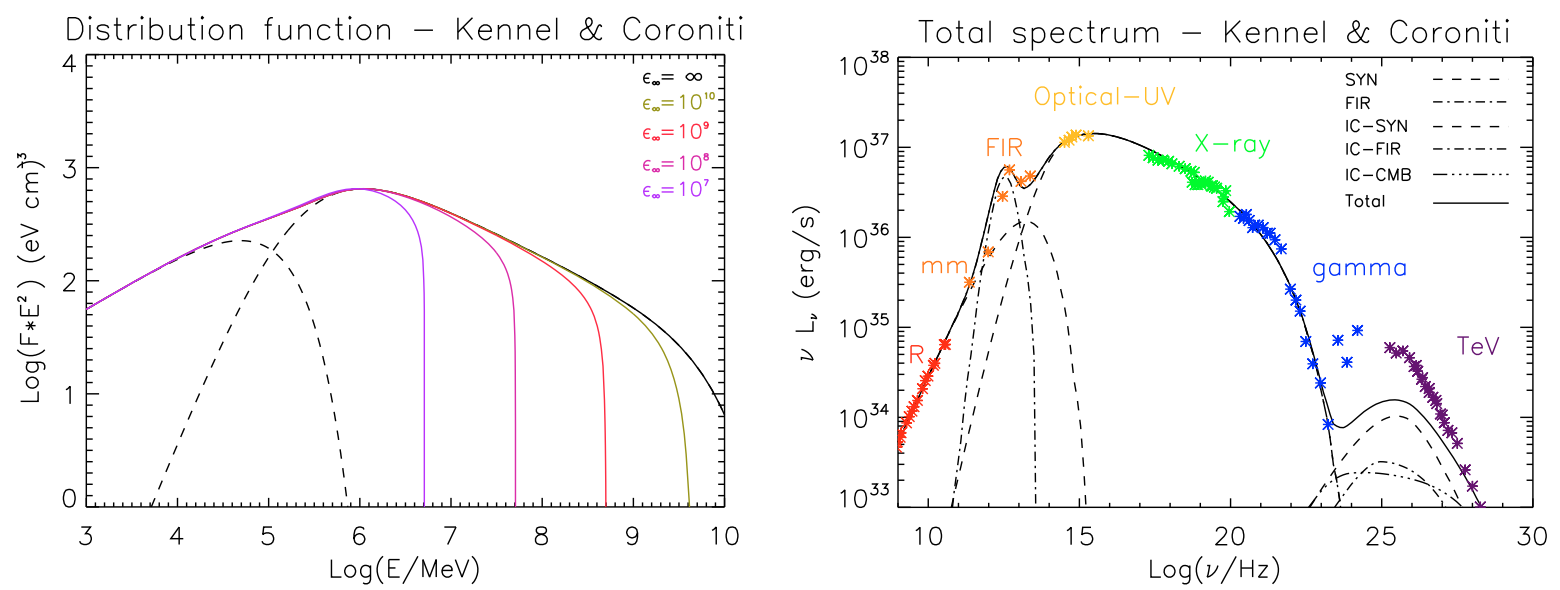

Fig. 1. Left panel: Kennel \& Coroniti total electron energy-distribution function for different values of the maximum particle energy $\epsilon_{\infty}$ as specified in the figure. In the plot, we use $E=\epsilon m c^{2}$ and $F=\left(f_{\mathrm{r}}+f_{\mathrm{w}}\right) \cdot 4 \pi /\left(m c^{2}\right)$ and the particle distribution function is normalized to an averaged pressure value of $2.1 \times 10^{-9} \mathrm{erg} \mathrm{cm}^{-3}$. The dashed lines are the primordial and the wind component at $\epsilon_{\infty} \rightarrow \infty$. Right panel: Kennel \& Coroniti spectra are plotted against the data. The single contributions from different incident photon targets, thermal FIR emission and total luminosity are reported with different colors and line-styles. Dashed lines: primordial and wind synchrotron contributions and IC-SYN emission; dash-dotted line: thermal FIR and IC-FIR ones; dash-dot-dotted line: IC-CMB one; solid line: total luminosity. References to the observations reported here are given in the main text.

can be as high as $30 \%$, on timescales of a few years, as we discuss later. Since this timescale is not much longer than the lightcrossing time of the inner nebula, the temporal lag between the emission of a photon and its comptonization, should be taken into account. This could be properly achieved using more complex, time-dependent, radiation-transfer equations, which should be solved simultaneously with the MHD dynamics. Such an analysis is beyond the goal of the present paper, where we assume an approximation of instantaneous comptonization. This implies that our results on gamma-ray variability, at frequencies that involve a time-varying photon target density, should be taken with caution.

In the case of the thermal FIR emission, the emitting dust is expected to be located in a torus created by the supernova progenitor and in optical filaments (Green et al. 2004). Since the dust volume and location are uncertain, we choose the crude approximation:

$n_{v}^{\mathrm{IC}-\mathrm{FIR}}\left(v_{\mathrm{t}}\right)=\frac{1}{c h v_{\mathrm{t}}} \frac{L_{v}^{\mathrm{FIR}}\left(v_{\mathrm{t}}\right)}{4 \pi R^{2}}$,

where $L_{v}^{\text {FIR }}$ corresponds to a uniformly-emitting region of blackbody radiation.

Finally, the CMB photon density is provided by the Plank distribution

$n_{v}^{\mathrm{IC}-\mathrm{CMB}}\left(v_{\mathrm{t}}\right)=\frac{8 \pi}{c^{3}} \frac{v_{\mathrm{t}}^{2}}{\exp \left(h v_{\mathrm{t}} / k_{\mathrm{B}} T\right)-1}$,

where $k_{\mathrm{B}}$ is the Boltzmann constant and $T=2.7 \mathrm{~K}$.

After the IC emission coefficient is calculated as a function of space, integration over the PWN volume provides the total spectral luminosity as in Eq. (11)

$L_{v}^{\mathrm{IC}}(v)=\int 4 \pi j_{v}^{\mathrm{IC}}(\boldsymbol{r}, v) \mathrm{d} V$,

where scattering with different targets is computed separately to investigate the relative contribution to the overall IC emission.

The surface-brightness maps with relativistic corrections are computed as for synchrotron emission.

\section{Integrated spectra: fitting the Crab Nebula data}

To understand the different contributions of the various physical processes to the overall emission of the Crab Nebula, simulated synchrotron, and IC integrated emission spectra are obtained and plotted against the data from the radio to the gamma-ray band.

Using this comparison, we derive the unknown parameters in the distribution functions, which were defined in the previous section, within two different dynamical models: the steady-state, spherically-symmetric model by Kennel \& Coroniti (1984a), used as a test, and our axisymmetric $2 \mathrm{D}$ simulations.

\subsection{The spherical model}

The emission recipes of Sect. 2 are applied to Kennel and Coroniti's spherical model, which is calculated for a windmagnetization parameter $\sigma=0.005$ (as did Atoyan \& Aharonian 1996) and a nebular radius $R=2.0 \mathrm{pc}$. Integrated spectra in Fig. 1 are compared with observations of the Crab Nebula and with the results by Atoyan \& Aharonian (1996), used here as a benchmark for our model.

The values of the parameters correspond to best-fit estimates (see Table 1). The primordial and wind spectral indices are equal to the observed values in the radio and optical bands (Baars \& Hartsuijker 1972; Véron-cetty \& Woltjer 1993), respectively. $A_{\mathrm{r}}$ and $A_{\mathrm{w}}$ are found by fitting the emission in the radio and $\mathrm{X}$-ray band; $\epsilon_{\mathrm{r}}^{*}$ and $\bar{\epsilon}$ are chosen to connect the radio and optical parts of the spectrum smoothly; $\epsilon_{\mathrm{w}}^{*}$ helps to reproduce the observations in the gamma-ray band. We recall that $A_{0}=A_{\mathrm{w}} p_{0}$ refers only to the non-thermal tail of the particle-energy distribution. This accounts for some unknown fraction $\xi$ of the thermal pressure at the termination shock (Kennel \& Coroniti 1984b), where $\xi$ is determined using:

$\xi p_{0}=\frac{m c^{2}}{3} \int_{4 \pi} \mathrm{d} \Omega \int_{\epsilon_{0}^{\min }}^{\epsilon_{0}^{\max }} f_{0}\left(\epsilon_{0}\right) \epsilon_{0} \mathrm{~d} \epsilon_{0} \quad 0<\xi<1$,

where $\Omega$ is the solid angle, $\epsilon_{0}^{\min }=\bar{\epsilon}, \epsilon_{0}^{\max }=\epsilon_{\mathrm{w}}^{*}$, and $f_{0}\left(\epsilon_{0}\right)$ contains $A_{0}$ and hence $p_{0}$. We find that $\xi \approx 70 \%$.

The parameter values are slightly different from those in the paper by Atoyan \& Aharonian (1996), from which the form of 
Table 1. Values of the parameters for the spherical model (KC) and for two different set up of our axisymmetric RMHD simulations, runA (1) and runA (2). $A_{\mathrm{r}}$ is in unit $\mathrm{cm}^{-3}$ and $A_{\mathrm{w}}$ is in unit $\mathrm{erg}^{-1}$.

\begin{tabular}{cccc}
\hline \hline Parameters & KC & RunA (1) & RunA (2) \\
\hline$\alpha_{\mathrm{r}}$ & 0.26 & 0.26 & 0.26 \\
$\alpha_{\mathrm{w}}$ & 0.70 & 0.70 & 0.85 \\
$A_{\mathrm{r}}$ & $2.90 \times 10^{-6}$ & $1.35 \times 10^{-5}$ & $1.35 \times 10^{-5}$ \\
$A_{\mathrm{w}}$ & $3.2 \times 10^{8}$ & $2.6 \times 10^{9}$ & $3.2 \times 10^{11}$ \\
$\epsilon_{\mathrm{r}}^{*}$ & $2.0 \times 10^{5}$ & $3.0 \times 10^{5}$ & $3.0 \times 10^{5}$ \\
$\epsilon_{\mathrm{w}}^{*}$ & $1.4 \times 10^{10}$ & $\infty$ & $\infty$ \\
$\bar{\epsilon}$ & $4.3 \times 10^{5}$ & $5.0 \times 10^{5}$ & $8.0 \times 10^{5}$ \\
\hline
\end{tabular}

our distribution function is taken. The main difference is in the cut-off energy $\epsilon_{\mathrm{w}}^{*}$ and is due to our approximated formula (5), where adiabatic losses are neglected (these are considered instead by means of the factor $p$ in Eq. (4)). However, the difference involves only the synchrotron spectrum at gamma-ray energies, with no consequences for the IC radiation flux, to which electrons close to the cut-off contribute negligibly (see also Fig. 6 and the discussion in Sect. 5).

In the left panel of Fig. 1, we plot the total evolved distribution function, given by Eqs. (1) and (4), for different values of $\epsilon_{\infty}$. The particle-distribution function is normalized using a value of the local pressure that corresponds to the volume average in the simulation. Since the relic population is assumed to be spatially homogeneous, the radial dependence is included only in the wind-particle distribution function using the local values of $p(r)$ and $\epsilon_{\infty}(r)$, both provided by the model of Kennel \& Coroniti (1984a,b). The wind population displays the expected behavior: both the local value of the thermal pressure, to which the peak of the distribution is proportional, and the cut-off energy, providing the exponential decay, are decreasing functions of $r$. Our results are basically coincident with those shown in Fig. 3 of Atoyan \& Aharonian (1996) in spite of a different visualization (see the respective captions).

The overall synthetic spectrum is calculated using the above distribution function and dynamical quantities from the Kennel \& Coroniti's model. In the right panel of Fig. 1, the spectral luminosity is plotted and the individual synchrotron contributions of the two populations are shown. The synchrotron spectrum from radio to IR frequencies is due to the primordial particles, homogeneously-distributed over the nebula. At higher frequencies, the emission is due to the wind particles, which suffer from adiabatic and synchrotron cooling. The FIR thermal radiation is obtained using the black-body formula for a temperature of emitting dust of $T=46 \mathrm{~K}$ (Strom \& Greidanus 1992; Green et al. 2004). As far as IC radiation is concerned, we find that the primordial electrons contribute to IC-CMB radiation up to $1.6 \times 10^{22} \mathrm{~Hz}$, to IC-FIR up to $3.2 \times 10^{23} \mathrm{~Hz}$ and to IC-SYN up to $7.9 \times 10^{23} \mathrm{~Hz}$ (in the paper by Atoyan \& Aharonian 1996, the frequency value is larger by almost one order of magnitude) mainly in the Thomson regime, $\epsilon h v_{\mathrm{t}} \ll m c^{2}$, while at higher frequencies, the emission is due to scattering by the wind particles.

References for the reported data are as follows: radio data are from Baars \& Hartsuijker (1972); the mm data are from Mezger et al. (1986) and Bandiera et al. (2002); the infrared points are from IRAS in the far to mid-infrared (Strom \& Greidanus 1992) and from ISO in the mid to near infrared range (Douvion et al. 2001); optical is from Véron-cetty \& Woltjer (1993) and UV from Hennessy et al. (1992). Points in the range between soft X and gamma-rays are taken from Kuiper et al. (2001), who compiled data from BeppoSAX, COMPTEL and EGRET. In the TeV band, we plot the data from MAGIC (Albert et al. 2008) and HEGRA (Aharonian et al. 2004).

Shapes and values of the single curves, especially IC-SYN and IC-CMB, are consistent with those of Figs. 9 of Atoyan \& Aharonian (1996). We therefore conclude that, in our model, the computation of inverse-Compton emission appears reliable. We recall that the main simplifications introduced are in the winddistribution function, Eq. (5), and the homogeneous emissivity in the IC-SYN target photon density, Eq. (18).

The computed synchrotron emission agrees with the data, whereas the IC luminosity is lower at all gamma-ray frequencies. In both the present $1 \mathrm{D}$ calculation and the model presented by Atoyan \& Aharonian (1996), the gamma-ray EGRET and $\mathrm{TeV}$ data can be simultaneously fitted only by invoking additional contributions to the emission namely Bremsstrahlung and hadronic processes. The magnetic-field strength can also be varied, although this has been attempted only in an ad hoc manner (Atoyan \& Aharonian 1996; Aharonian et al. 2004), rather than changing the dynamics self-consistently.

The conclusion that the IC alone is insufficient to explain the gamma-ray fluxes from the Crab Nebula appears to be the common result of all 1D models. However, 1D stationary models are not able to account for a number of observed nebular properties, in particular the jet-torus morphology observed in the $\mathrm{X}$-rays. Due to the oversimplified dynamics assumed by spherical models, the conclusions about the emission processes are also questionable. On the other hand, 2D time-dependent RMHD models have reproduced the inner structure of the Crab Nebula, even in some of its finest details. In the following sections, we present a study of the synchrotron emission and, for the first time, IC gamma-ray radiation from PWNe, in the context of the axisymmetric scenario.

\subsection{Results from numerical simulations}

\subsubsection{General discussion}

For the study of the non-thermal emission in the context of a 2D model, we focus on the simulation labelled as runA in the paper by Del Zanna et al. (2006). The parameters that determine the shape and the strength of the magnetic field are: the anisotropy, $\alpha=0.1$, the width of the striped wind region, $b=10$ (corresponding to $\approx 10^{\circ}$ around the equator), and the wind magnetization, $\sigma=0.025$. The maximum energy of the particles at injection, $\epsilon_{\infty}$, is taken to be $10^{10}$, which corresponds to $5 \times 10^{15} \mathrm{eV}$ (see e.g. De Jager \& Harding 1992; Aharonian et al. 2004).

We discuss the nebular emission spectra that are produced by our simulation. Two different distribution functions are considered for the wind particles: one with a wind spectral index of $\alpha_{\mathrm{w}}=0.7$, runA (1), and another with $\alpha_{\mathrm{w}}=0.85$, runA (2). The first is the counterpart of the spherical model computed in the previous section, which corresponds to the best-fit optical wind spectral index (Véron-cetty \& Woltjer 1993). Using this value of $\alpha_{\mathrm{w}}$, we are unable, however, to fit the synchrotron spectrum from X-ray to gamma-ray frequencies. Furthermore, the model IC emission clearly exceeds that of the data, as can be observed in the right panel of Fig. 2. A second steeper injection spectrum is therefore adopted to ascertain how the combination of synthetic synchrotron and IC emission can be adapted to determine the physical parameters of PWNe. This second spectrum does reproduce accurately the energy synchrotron spectrum (see Fig. 3) although its $\mathrm{TeV}$ emission is slightly closer to, but still stronger than that of the data. In both runs, the spectrum about $10 \mathrm{GeV}$ appears to fit the EGRET data approximately, that, we recall, 

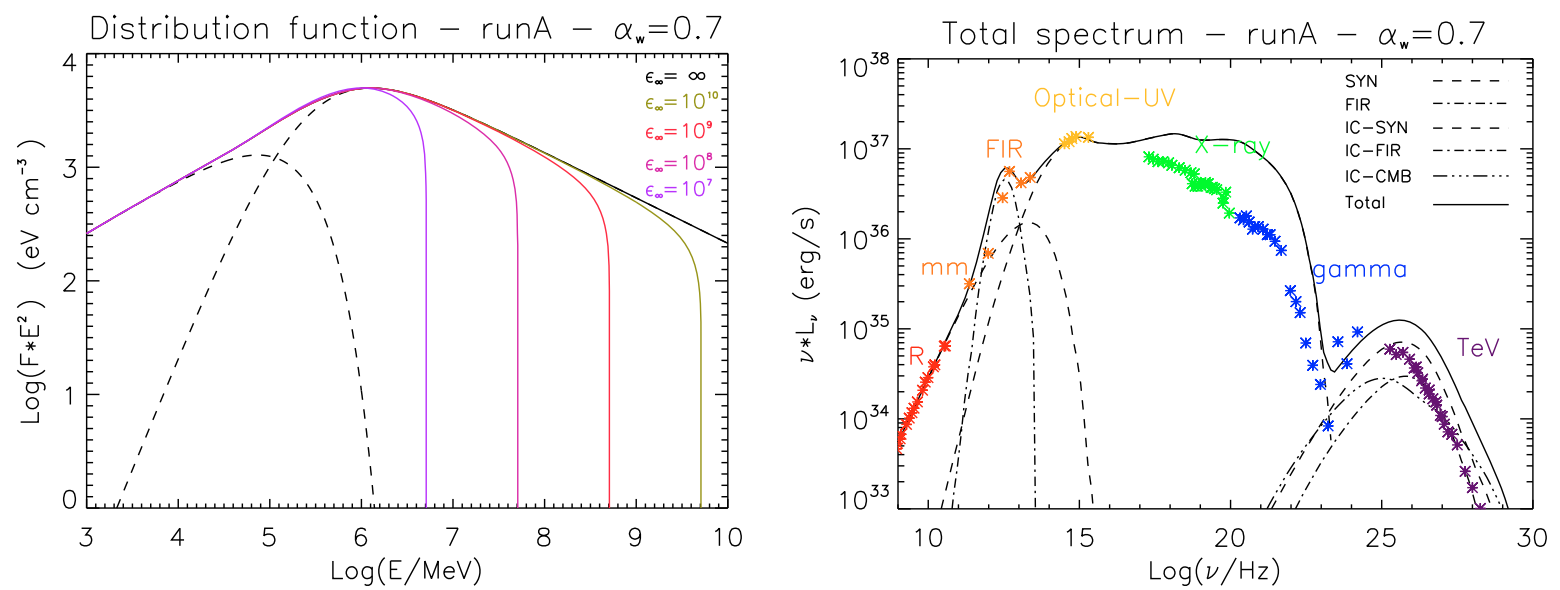

Fig. 2. Left panel: runA (1) total electron energy distribution function versus particle energy for different values of maximum particle energy $\epsilon_{\infty}$ as specified in the figure. In the plot, we use $E=\epsilon m c^{2}$ and $F=\left(f_{\mathrm{r}}+f_{\mathrm{w}}\right) \cdot 4 \pi /\left(m c^{2}\right)$ and the particle distribution function is normalized to an averaged pressure value of $2.1 \times 10^{-9} \mathrm{erg} \mathrm{cm}^{-3}$. The dashed lines are the primordial and the wind component for $\epsilon_{\infty} \rightarrow \infty$. Right panel: runA (1) spectra are plotted against the data. The single contributions from different incident photon targets, thermal FIR emission, and total luminosity are reported with different colors and line-styles. Dashed lines: primordial and wind synchrotron contributions and IC-SYN emission; dash-dotted line: thermal FIR and IC-FIR ones; dash-dot-dotted line: IC-CMB one; solid line: total luminosity. References to the observations reported here are given in the main text.
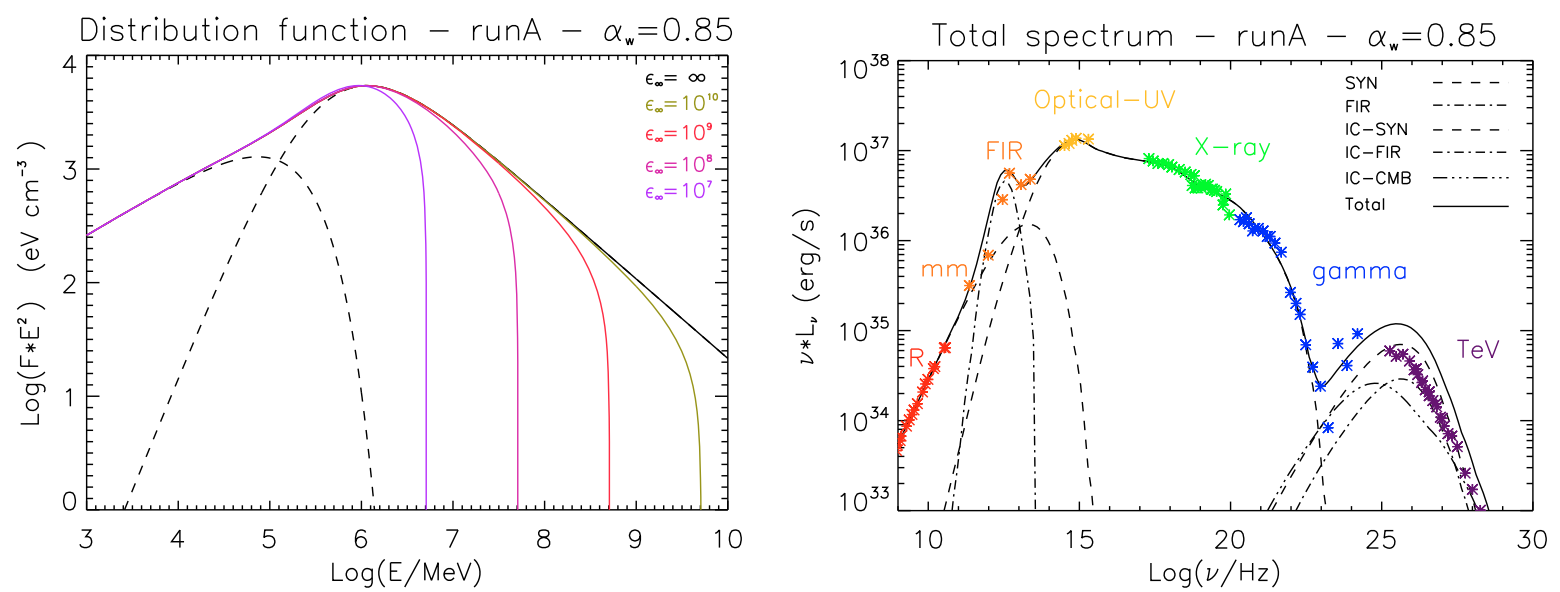

Fig. 3. The same as Fig. 2 for runA (2).

are affected by large error bars (Kuiper et al. 2001). Hopefully, GLAST observations will enable the models to be more accurately constrained for this crucial range of frequencies. At the moment, the additional contributions to the gamma-ray emission, such as Bremsstrahlung and hadronic processes, cannot be accommodated by the models. We note that these results are the inverse of those found for 1D models (see Sect. 3.1): in that case, the model underpredicts the IC peak emission by a factor of approximately five.

Before discussing the spectra in detail, we first describe the particle distribution function. The total (primordial plus wind) particle distribution functions in both cases, which were obtained as described in Sect. 2.1, are shown in the left panels of Figs. 2 and 3. The values adopted for the maximum electron energy $\epsilon_{\infty}$ and the thermal pressure, are similar to those used in Fig. 1. However, we should remember that these quantities are functions of both spherical radius $r$ and polar angle $\theta$ (see Fig. 4). The remaining parameters are chosen so that the observations are reproduced as successfully as possible by our simulated spectra (see Table 1).

By comparing the left panels of Figs. 2 and 3 with the corresponding panel of Fig. 1, we see that, in runA (1) and runA (2) simulations, there is a larger number of electrons present at all energies compared to the spherical case. However, in runA (2), the distribution function decreases rapidly at high energies, reaching values that are close to those found within the spherical model.

The values of the parameters adopted are reported in Table 1. We note that for the assumed injection energy, $\epsilon_{\infty}$ never exceeds $10^{10}$. Hence, the synchrotron cut-off in gamma-rays is reproduced correctly, without the need to introduce an exponential decay of the particle distribution function.

For both cases, runA (1) and runA (2), the emission spectrum is calculated, as described in Sect. 2, and displayed in the right panels of Figs. 2 and 3. As far as the different contributions of the two populations to the different frequency bands is concerned, the primordial electrons contribute to synchrotron emission up to IR frequencies, to IC-CMB emission up to $2.5 \times 10^{22} \mathrm{~Hz}$, to IC-FIR up to $3.2 \times 10^{23} \mathrm{~Hz}$, and to IC-SYN up to $7.9 \times 10^{23} \mathrm{~Hz}$, mainly in the Thomson regime. The radiation at higher frequencies, for all components, is due instead to wind electrons. In both runs, the data are reproduced well in the radio (by construction), and in the optical bands. In this context, the first spectral break, between the IR and optical, appears to be intrinsic and due to the 

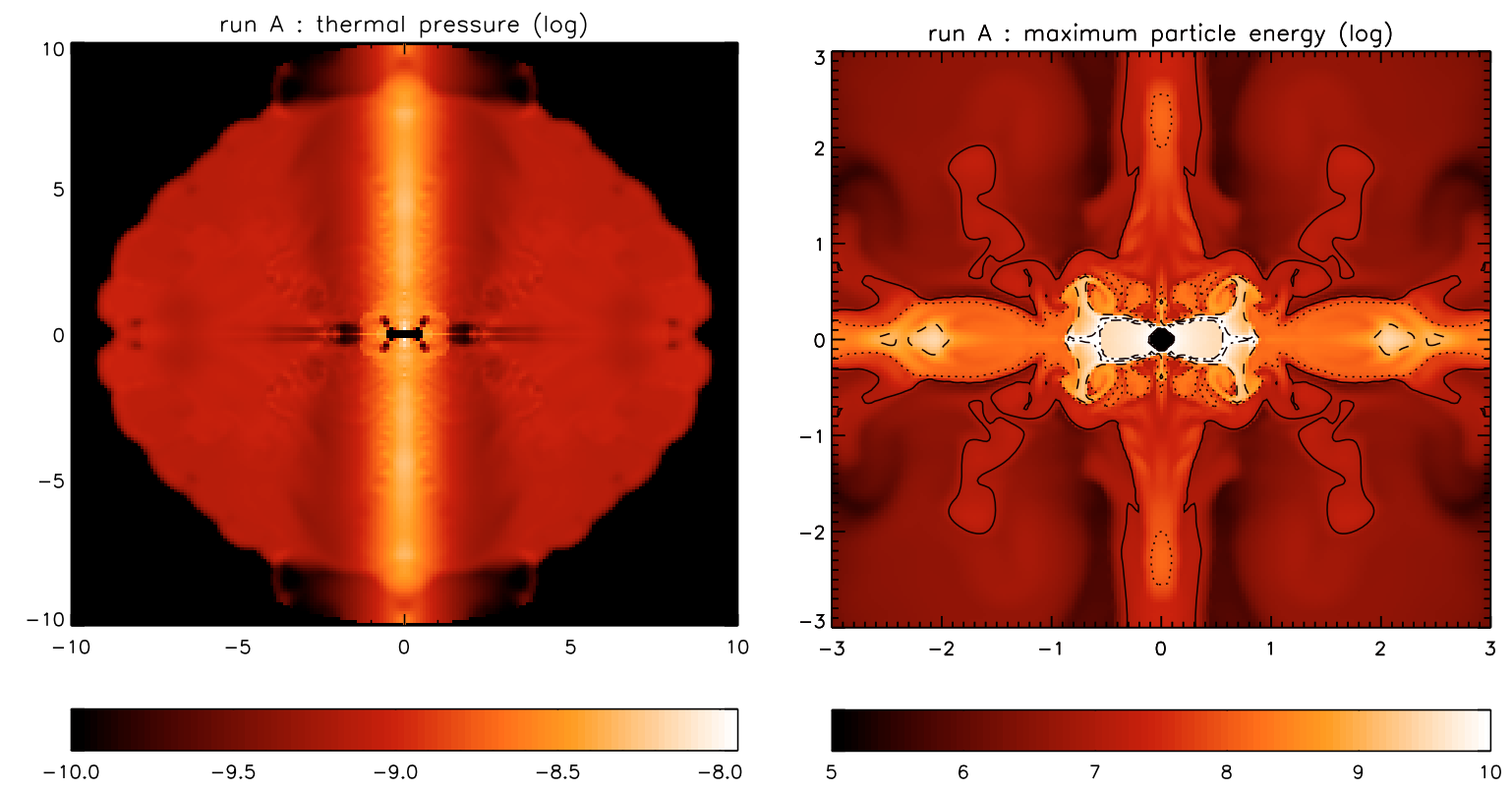

Fig. 4. Left panel: color-coded map of the thermal pressure in units of $\mathrm{erg}^{-3}$. Right panel: color-coded map of the maximum particle energy; superimposed contours correspond to values of $10^{7}$ (solid line), $10^{8}$ (dotted line), $10^{9}$ (dashed line), and $10^{10}$ (dot-dashed line). Along the axes, distance from the central pulsar is reported in ly.

superposition of two different populations of particles. The second spectral break, in the UV band, corresponds, in our model, to a strong synchrotron burn-off and is related to the decreasing volume occupied by particles of increasing energy. Beyond $\approx 2 \times 10^{15} \mathrm{~Hz}$, we observe a spectral flattening in our curves, which is followed by further minor breaks and counter-breaks at higher frequencies. We recall that Kargaltsev \& Pavlov (2008) presented X-ray observations of PWNe with spectral indices less than 0.5 , which had no theoretical explanation besides that the data could be reproduced in principle, by simulations similar to runA (1).

In the case of runA (1), the computed synchrotron emission at frequencies above $\approx 10^{16} \mathrm{~Hz}$ is far in excess of the data. This is also the case at frequencies higher than $\approx 10^{24} \mathrm{~Hz}$, where inverse Compton is the dominant emission mechanism. We note, however, that the IC-SYN component is relatively unaffected by the hard X-ray and gamma-ray synchrotron excess, which contributes only beyond $1 \mathrm{TeV}$. In runA (2), we manage to fit the $\mathrm{X}$-ray data using our steeper simulated spectra, but the IC emission still appears to exceed the data by a factor $\sim 2$.

These results imply that to reproduce the synchrotron spectrum, we need to change more than the particle-distribution function, and, specifically, than the commonly-accepted value of $\alpha_{\mathrm{w}}$; the underlying dynamics may need to be revised, in particular the nebular magnetic-field distribution.

In our simulations the nebular magnetic field appears to be compressed in localized regions close to the termination shock, and decrease rapidly outward, much faster than in spherical models. Its volume-averaged value is $B_{\text {mean }}=1 \times 10^{-4} \mathrm{G}$, which is $2-3$ times less than the value predicted by previous models (Kennel \& Coroniti 1984a). The outer regions occupy a large fraction of the volume and contribute in a way proportional to its volume, to the integrated emission; our synthetic magnetic field in the outer regions is therefore much lower than for spherical models. To be able to fit the integrated spectra, we had to introduce a larger number of emitting electrons than for the spherical model. Our electrons are affected by lower synchrotron losses, on average; a steeper spectral index $\alpha_{\mathrm{w}}$, as in runA (2), is therefore required to reproduce the high-energy synchrotron spectrum. The simultaneous calculation of the IC spectrum, however, indicates that this required large number of emitting electrons then causes disagreement with the gamma-ray data. Therefore, to reproduce the spectrum of the Crab Nebula, a larger and more extended magnetic field is required, which should also reproduce the correct dynamics. Before drawing conclusions on the physics of the emission processes, a further investigation of the parameter space (for both the dynamics and the particle distributions) should be, however, attempted, since we considered only runA results. The problem of the concentration of the nebular magnetic field is possibly an intrinsic problem of axisymmetric simulations that could only be resolved by moving to $3 \mathrm{D}$.

\subsubsection{On the spectral slopes}

We return to the most remarkable features observed in our spectra, the multiple changes of slope, which conflict with the simple expectations of synchrotron cooling. However, the prediction of one single steepening of the slope due to synchrotron burn-off is based on simplified 1-zone or 1D nebular models, which assume a smooth flow and magnetic field distribution, such that particles experience continuous synchrotron losses. In contrast, one of the principle results of 2D MHD models of PWNe was that the internal-flow dynamics are more complex than previous 1D models had suggested.

This complexity is reflected in the particle spectrum, as a function of position inside the nebula. This is computed as described in Sect. 2.1, using information about the thermal pressure $p$ and the maximum energy $\epsilon_{\infty}$ that are provided by the simulations: color-coded maps of $p$ and $\epsilon_{\infty}$, are shown in the left and right panel of Fig. 4, respectively.

The computed pressure converges to a constant value and has a spatial average of $2.1 \times 10^{-9} \mathrm{erg} \mathrm{cm}^{-3}$, which is behind our choice of normalization constant in plots that show the population distribution functions in Figs. 1-3. Exceptions are observed along the jets, where the maximum is located, and in the equatorial plane, where vortices create regions of lower pressure. 
As far as the local value of maximum energy is concerned, the effects of synchrotron burn-off are highlighted by the four contours at the top of the color-coded map of $\epsilon_{\infty}$. These contours correspond to the same values as that considered for the particle distribution functions, shown in the left panels of Figs. 1-3. The main difference between the results of our 2D simulations and spherically-symmetric models, is immediately apparent from the contours in the right panel of Fig. 4: the spatial domain occupied by particles of energies up to a certain value, consists of disconnected regions. In 1D, the contours would be connected and encircle progressively smaller regions of space with increasing values of $\epsilon_{\infty}$. In contrast, the flow structure and magnetic-field distribution is such that this is no longer the case. In our simulations fast-flow channels, in regions of relatively low magnetic field, may transport high-energy particles to significant distances from the termination shock, without experiencing significant synchrotron losses. The spatial distribution of the magnetic field produced by our model, which is compressed by the flux vortices around the TS and polar axis (see Del Zanna et al. 2004, 2006), causes, in addition, a non-monotonic decline of the volume occupied by high-energy particles. This is the origin of the multiple changes of slope in the synthetic emission spectrum. We note that 2D simulations completed using a lower value of the magnetization parameter produce far less structured flow patterns and spectra with one change of slope only, which are analogous to those found from spherical models. At the same time, however, the jets disappear. Based on the possibility that we find here of more than one spectral break being created due to synchrotron losses, we speculate that a larger value of the magnetization parameter, which is sufficient to move the multi-slopes to IR frequencies, could help reproduce the complete synchrotron spectrum of the Crab Nebula, using only one population of particles. Additional particle acceleration may occur, however, in regions other than the termination shock; in particular, it can occur at the termination spots of high-speed flows, in the equatorial plane, and along the jets, and cause changes in slope.

\subsubsection{On the SYN/IC estimate for $B_{\text {mean }}$}

The ratio between synchrotron and IC observed luminosity, when these data are available, is often used to evaluate the average magnetic-field strength in PWNe (e.g. De Jager et al. 1996; HESS Collaboration: Djannati-Atai et al. 2007, and references therein). However, this estimate is based on the assumption that the magnetic field is uniformly distributed (or slowly varying as in the $\mathrm{KC}$ model). On the other hand, numerical results of PWNe have shown clearly (as also confirmed in this paper, see the discussion in this section) that the distribution of the magnetic field and the maximum energy of the emitting particles, can change quite dramatically within the nebula.

It is of interest to theorists, not only observers, to understand if, in the case of 2D PWNe models, the magnetic field inferred from the ratio SYN/IC provides a good approximation of the actual value, or is biased, at different frequencies.

In particular, we compare the estimate of the magnetic field from the ratio SYN/IC, computed using the synthetic spectra, with the average value of the magnetic field $B_{\text {mean }}$ equal to $1 \times 10^{-4} \mathrm{G}$ in our RMHD simulations, on which the same spectra are based. The measured discrepancy is approximately $10 \%$ at synchrotron frequencies below $10^{23} \mathrm{~Hz}$, while beyond $10^{28} \mathrm{~Hz}$, the discrepancy increases to larger than a factor of 2 . This is because, in the high frequency range, the emission originates in wind particles, confined to a small volume around the TS, where the magnetic field is highly structured and higher than the average value.

\section{Gamma-ray surface brightness maps}

Synthetic IC surface-brightness maps are shown, for the first time, in the gamma-ray band. These are calculated for runA (2) by integrating along the line of sight, the total (IC-SYN, IC-FIR and IC-CMB) emissivity, where Doppler relativistic effects are included as described by Del Zanna et al. (2006).

In Fig. 5, images at photon energies of $4 \mathrm{GeV}, 250 \mathrm{GeV}$, and $1 \mathrm{TeV}$ are computed. These are chosen to be at the center of the band that will be observed by GLAST $(20 \mathrm{MeV}-300 \mathrm{GeV})$, in the range measured by MAGIC $(60 \mathrm{GeV}-9 \mathrm{TeV})$, and at the central energy of the HESS band $(100 \mathrm{GeV}-10 \mathrm{TeV})$. When comparing the three maps, it is immediately apparent that the size of the nebula is larger at lower frequency.

The second feature is that the jet-torus structure, which is common among the observed PWNe in the X-rays and produced by the simulated RMHD evolution in time and space with a $\sigma_{\text {eff }} \gtrsim 0.01$, is clearly visible also at gamma-ray frequencies. We can distinguish the bright features as the central knot and the arcs, although the brightness contrast is now less than in the X-rays (see Del Zanna et al. 2006). This similarity between the gamma-ray and X-ray morphology is due to the fact that the local emitting electrons are the same particles for both bands.

A study of these bright features in our synthetic surfacebrightness maps at various synchrotron frequencies showed that the central knot and arcs close to the TS, appear at all energies and completely dominate the gamma-ray emission. Larger structures such as the jets and the torus, begin to fade in images at $\gtrsim 1 \mathrm{keV}$ and $\approx 10 \mathrm{keV}$, respectively. Rising in frequency from the synchrotron peak the overall PWN size, continues to decrease, reaching a minimum at the cut-off SYN frequency $(0.5 \mathrm{GeV}$ in our model), where basically only electrons at the TS contribute. The original (radio-band) dimensions are recovered, when IC becomes the dominant emission process, due to the ubiquity of the radio electrons responsible for emission at $\mathrm{GeV}$ frequencies. When the wind electrons begin to dominate the emission, synchrotron burn-off effects start to play a role and the volume of the nebula decreases again. All structures, that disappear in the hard X-ray synchrotron emission are visible in gamma-ray images up to $\mathrm{TeV}$ photon energies. This is because the IC emission structure does not directly depend on the complex magnetic-field distribution, but only on the spatial behavior of the electron distribution function, which is determined by the local value of $p$ and $\epsilon_{\infty}$.

Present-day gamma-ray instruments have insufficient spatial resolution to distinguish single features in the internal structure, so that a comparison between the simulations and the data can be based only on the size of the nebula. However, indications of asymmetries in PWNe are already seen by HESS (Aharonian et al. 2005b, 2006a).

MAGIC measured an average radius of about $4.5 \mathrm{ly}$ at $250 \mathrm{GeV}$ and of about $3 \mathrm{ly}$ at $500 \mathrm{GeV}$ (Albert et al. 2008). The simulated maps at the corresponding frequencies (we do not display here the $500 \mathrm{GeV}$ image) have dimensions comparable to the observed values along the $y$-axis, although along the $x$-axis, we find a negligible shrinking of the size with growing frequency. This is due to the combined effect of our relatively low magnetic field and the presence of fast flow channels in the equatorial region, which advect particles outward fast enough for energy losses to be negligible. The average IC fluxes, observed by MAGIC, are about half of those obtained in our 

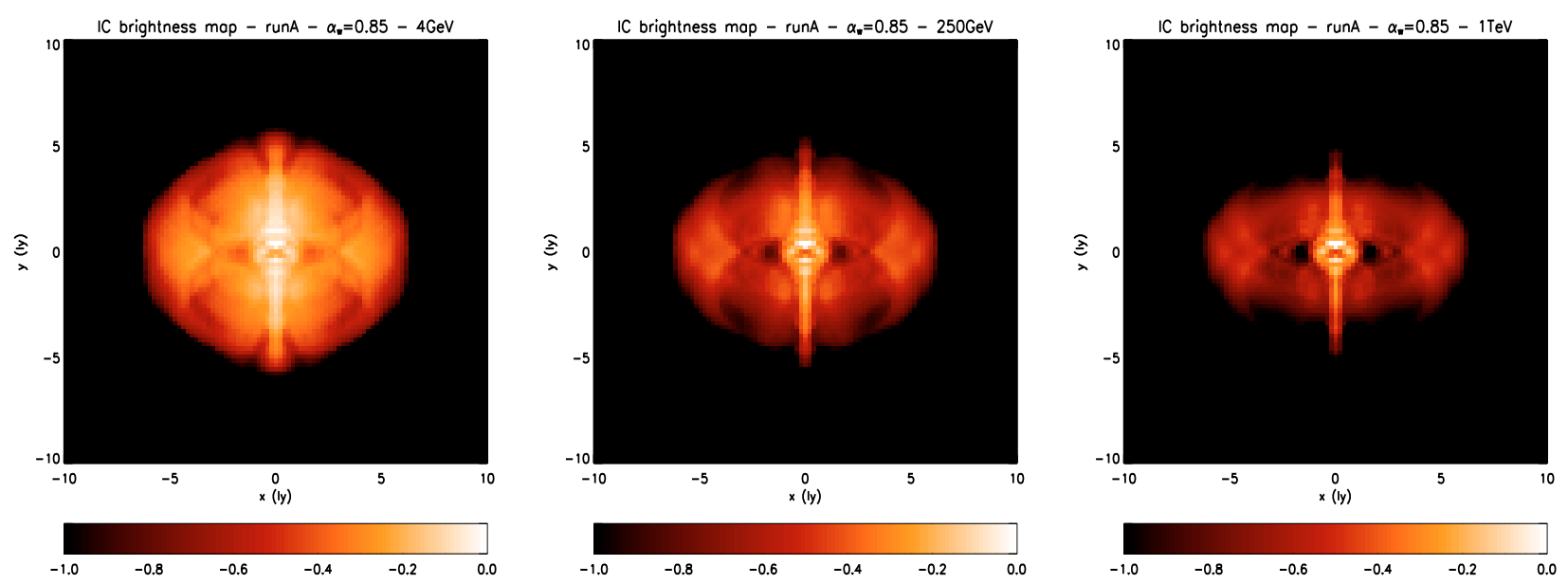

Fig. 5. RunA (2) case. Simulated total surface-brightness maps in logarithmic scale normalized to the maximum at different IC fre-

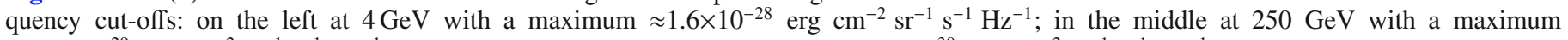
$\approx 1.0 \times 10^{-29} \mathrm{erg} \mathrm{cm}^{-2} \mathrm{sr}^{-1} \mathrm{~s}^{-1} \mathrm{~Hz}^{-1}$; on the right at $1 \mathrm{TeV}$ with a maximum $\approx 1.8 \times 10^{-30} \mathrm{erg} \mathrm{cm}^{-2} \mathrm{sr}^{-1} \mathrm{~s}^{-1} \mathrm{~Hz}^{-1}$. On the axes: distance from the central pulsar in unit of ly.

simulations $\left(\approx 4 \times 10^{-10} \mathrm{erg} \mathrm{cm}^{-2} \mathrm{~s}^{-1}\right)$. This corresponds to the excess of synthetic IC emission already discussed, when analyzing the spectra in Sect. 3.2.

\section{X-ray and gamma-ray time variability}

Bright variable emission features are observed in a few PWNe, including the Crab Nebula, at small scales close to the termination shock and in the torus. In the first case, they are called wisps, in the second filaments of the torus. The variability timescale is of kilo-seconds in the IR band (Gemini North Telescope, Melatos et al. 2005), from days up to a few months, and from months up to a year, at optical and X-ray frequencies (HST and Chandra, Weisskopf et al. 2000; Hester et al. 2002), with timescales that become longer with increasing distances from the TS. Quasi-periodically, these structures appear, brighten very quickly, and eventually fade, showing a characteristic outward motion. There are no corresponding gamma-ray observations because of the insufficient spatial resolution at those frequencies.

Different interpretations were proposed to explain these time-variable features. A kinetic interpretation was given by Spitkovsky \& Arons (2004), who showed that if protons are present in the pulsar wind, the variable structures can arise from ion-cyclotron instability at the shock front. Alternative explanations, in the context of ideal MHD, are based on nonlinear Kelvin-Helmholtz instabilities inside the nebula (Begelman 1999; Bogovalov et al. 2005; Bucciantini \& Del Zanna 2006; Bucciantini et al. 2008). Bucciantini (2008) presented simulations in which wisps close to the TS move outward at velocities of $\sim 0.5 \mathrm{c}$, and of slower filamentary structures in the torus, as observed. The period of the variability is approximately one year, which agrees with results for Chandra X-ray observations. We are aware that Bucciantini and Komissarov are carrying out a detailed study of time variability within MHD models, to understand its origin, its characteristics, and possible correlations with other nebular properties. Without going into detail, we investigate if time-dependent flow dynamics, responsible for X-ray variability, can also lead to fluctuations in the gamma-ray emission (De Jager et al. 1996; Ling \& Wheaton 2003) that might be of interest for future instruments (i.e. GLAST).

We consider runA (2) and compute snapshots of the integrated spectrum, synchrotron surface-brightness map at $1 \mathrm{keV}$
(Chandra band), and the IC surface-brightness map at $250 \mathrm{GeV}$, which is in the middle of the gamma-ray band observed by MAGIC, and towards the high-energy limit of, but still within, the GLAST band. The sequence of images lasts ten years, from $950 \mathrm{yr}$ to $960 \mathrm{yr}$, with intervals of $0.2 \mathrm{yr}$. A selection of snapshots of the movie is shown in Fig. $6^{1}$.

Our simulations confirm the presence of variable wisps close to the TS and filamentary structures (rings) in the inner part of the torus, with characteristic timescales of about $1-2$ years (as observed for energies less than 0.75 MeV, Much et al. 1995). These features move at speeds $0.3-0.5 \mathrm{c}$ (see also the flux velocity maps in Del Zanna et al. 2006), and decelerate before fading out at larger distances, which agrees with observations. This result suggests that variability in the inner nebula can be interpreted as being associated with fluid motions that occur in the vicinities of the TS (either MHD compressive modes or KelvinHelmholtz instabilities, the latter arising at the shear flow boundaries). Apart from these time-varying features, the most prominent structures, namely the central knot and the main arc, are instead stationary, as observed.

The synthetic synchrotron surface-brightness maps at X-ray frequencies, and IC emission maps at $250 \mathrm{GeV}$ photon energies, show a strongly-correlated variability, which is due to motion of the common parent electrons. Since the IC emission is more uniformly distributed in the nebula than high-energy synchrotron emission from the same electrons, the small-scale moving features are, however, less prominent than in X-ray maps, as expected. The variability of the integrated IC spectrum is correspondingly reduced. We recall that in our model at photon energies up to $1 \mathrm{TeV}$, the IC emission is found to be due to wind electrons up-scattering radio-IR photons. A study of time-variability in this band, therefore, still makes sense, in spite of the simplified assumptions that underlie our computation of the IC emission. We recall that we neglect all propagation effects in space and time, by approximating the energy density of the target photon field to be uniform over the nebular volume and slowly varying. Both of these assumptions are satisfied in the radio-IR band.

For a more quantitative analysis of the variability properties we show, in Fig. 7, the synthetic integrated emission as a

\footnotetext{
1 The full movie is downloadable from the electronic edition of A\&A, or from http://www . arcetri .astro.it/ delia/crab/ runA2.gif
} 

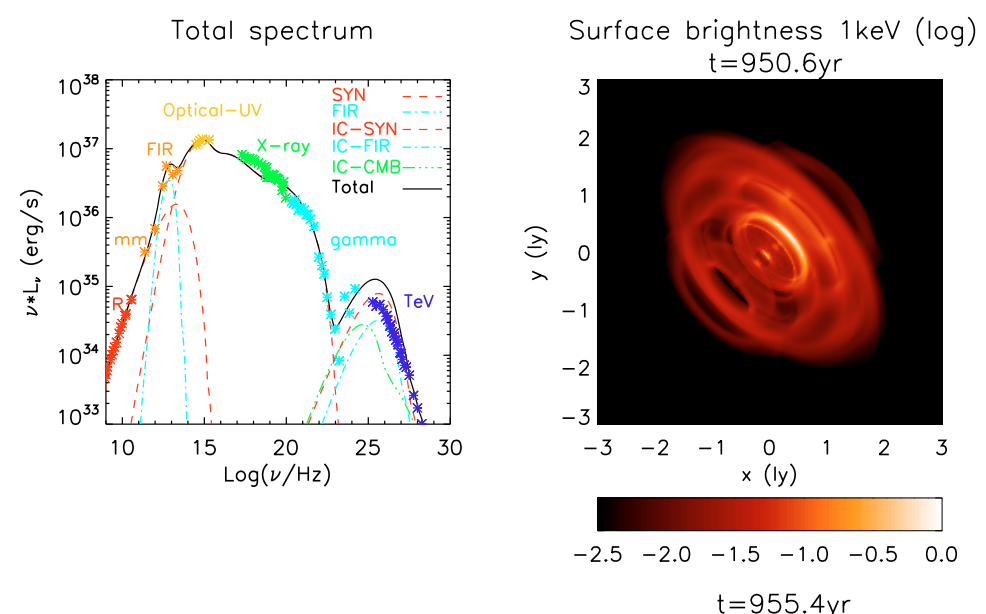

Surface brightness $250 \mathrm{GeV}$ (log)
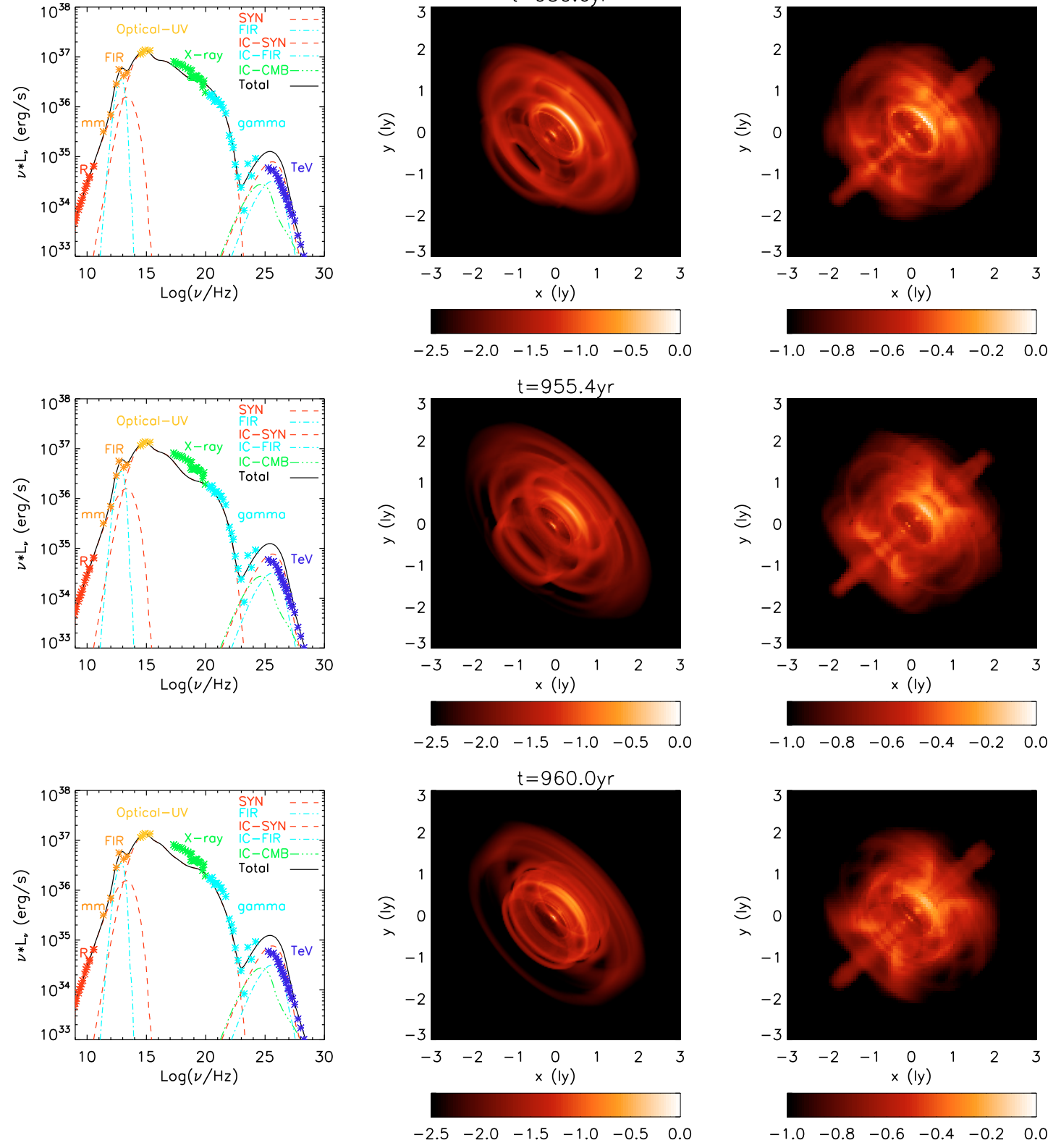

Fig. 6. RunA (2) case. Snapshots from a movie of PWN evolution: output times are selected at $950.6 \mathrm{yr}, 955.4 \mathrm{yr}$, and $960.0 \mathrm{yr}$. Results from one output time per row are shown. Left panels: plot of the synthetic spectral luminosity in logarithmic scale versus photon frequencies (log). Middle panels: simulated brightness maps (log) at $1 \mathrm{keV}$ normalized to the maximum $6.2 \times 10^{-18} \mathrm{erg} \mathrm{cm}^{-2} \mathrm{sr}^{-1} \mathrm{~s}^{-1} \mathrm{~Hz}^{-1}$. Right panels: simulated brightness maps (log) at $250 \mathrm{GeV}$ normalized to the maximum $1.2 \times 10^{-29} \mathrm{erg} \mathrm{cm}^{-2} \mathrm{sr}^{-1} \mathrm{~s}^{-1} \mathrm{~Hz}^{-1}$. The $x$ and $y$ axes of all surface brightness maps report the distance (in ly) from the central pulsar. We indicate the inclination with respect to both the plane of the sky and the north direction: respectively $30^{\circ}$ and $48^{\circ}$ for the Crab Nebula. We note that only the internal region (within a radius of 3 ly) is displayed.

function of time, for a selection of photon energies from X-rays to $\mathrm{TeV}$ gamma-rays ( $1 \mathrm{keV}, 40 \mathrm{MeV}, 1 \mathrm{TeV})$. The strongest variations (about a factor of 2) are observed at synchrotron gammaray frequencies, where the emission is due entirely to the moving features close to the TS. On the other hand, the IC time series show very limited (about $1 \%$ ) variations, as anticipated above. The results at synchrotron frequencies agree with observations by De Jager et al. (1996) who measured flux variations in the $1-150 \mathrm{MeV}$ band, finding an amplitude of $30 \%$ in the COMPTEL region (1-30 MeV), and a factor of 2 in the EGRET band $(70-150 \mathrm{MeV})$.

As far as timescales are concerned, the gamma-ray variability, with a lower limit of approximately a few years in our simulations, agrees with the results by De Jager et al. (1996). However, the clear relationship between the moving features observed in the simulated surface-brightness maps of Fig. 6, cannot be easily recovered in the luminosity time series, due to spatialintegration effects. In any case, a combination of XMM/Chandra and GLAST observations might confirm the similarities between $\mathrm{X}$-ray and gamma-ray variability found in our simulations, and consequently prove their common MHD origin.

\section{Conclusions}

We extend the modeling of Del Zanna et al. (2006), on synchrotron emission from 2D RMHD simulations of the PWNe 


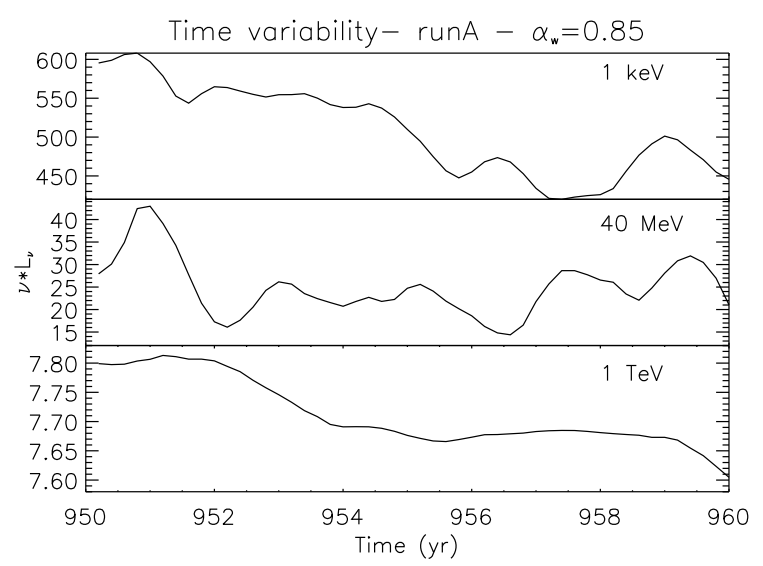

Fig. 7. RunA (2) case. Total luminosity $v L_{v}$ in unit $10^{34} \mathrm{erg} \mathrm{s}^{-1} \mathrm{~s}$ is displayed as a function of time, from $t=950 \mathrm{yr}$ to $t=960 \mathrm{yr}$. From top to bottom panels, the corresponding photon energies are $1 \mathrm{keV}, 40 \mathrm{MeV}$, $1 \mathrm{TeV}$.

(in particular, the Crab Nebula), to the gamma-ray frequencies, including the calculation of inverse Compton emission self-consistently. For the first time, maps of IC surface brightness are computed, which are compared with existing (e.g. COMPTEL, HEGRA, HESS) and future observations (GLAST) of the Crab Nebula. Following studies in soft X-ray wavelengths (Bucciantini 2008), an investigation of time variability in the gamma-ray band is presented.

To compute the IC emission, we include, following Atoyan \& Aharonian (1996), a homogeneously-distributed population of radio-emitting electrons that we assume form during the outburst of the $\mathrm{SN}$, in addition to the wind electrons, which are continuously accelerated at the TS. In this scenario, the spectral break between IR and optical frequencies $\left(\approx 10^{13} \mathrm{~Hz}\right)$ is intrinsic and due to the superposition of the two populations, while synchrotron burn-off produces the spectral break in the $\mathrm{UV}\left(\approx 2 \times 10^{15} \mathrm{~Hz}\right)$.

By comparing our 2D RMHD simulations with the Crab Nebula integrated spectra in Sect. 3.2, we observe that the value $\alpha_{\mathrm{w}}=0.7$, which was suggested by the optical data of the inner parts of the nebula (Véron-cetty \& Woltjer 1993) and adopted by Atoyan \& Aharonian (1996), overestimates the synchrotron emission at frequencies above $10^{16} \mathrm{~Hz}$. It is possible only with the spectral index $\alpha_{\mathrm{w}}=0.85$ to reconcile the simulated emission with high-energy synchrotron data of the Crab Nebula. However, the computed IC emission exceeds the observed gamma-ray data flux by a factor of 2, which indicates that the steeper particle spectrum is insufficient to remove the discrepancy between the results of the simulations and the data. The problem is related to the magnetic field in the simulations, which is compressed towards the termination shock and lower on average than estimates found in the literature; furthermore, it is probably lower in value than the appropriate magnetic-field strength of the Crab Nebula. To reproduce the observed data, we must therefore consider a larger number of particles. This appears to resolve the problems so long as only synchrotron emission is considered; its limitations, however, are clearly evident when we consider in addition the calculation of IC. This appears to imply that a higher value of the pulsar wind magnetization should be adopted; we caution, however, that it is unclear how this would affect the nebular morphology, which is at present well reproduced. We find, in addition, that the complex nebular dynamics, highlighted by our 2D simulations, produces multi-slopes in the simulated synchrotron spectra, which are not expected to occur in 1D models.
Further insight can be gained from the gamma-ray morphology. Our synthetic maps indicate the nebular dimensions and the reduction in the size with increasing frequency, in the range of those observed by MAGIC (Albert et al. 2008), only along the polar axis, while dimensions do not change appreciably along the equator. This is due to fast-flow channels that develop in a region of low magnetization: these advect electrons far from the termination shock with negligible synchrotron losses.

The simulated IC surface-brightness maps are almost identical to the synchrotron X-ray maps, since they are produced by the same parent electrons. However, structures such as the jets and the external torus, which disappear in hard X-rays, are visible at the entire range of gamma-ray frequencies due to the fact that the emission is not directly affected by the local magnetic field.

Both the X-ray and gamma-ray variability of the inner nebula appear to have MHD origins. In our simulations we find characteristic timescales of about $\approx 1-2$ yr for both types of variability. We find, in addition, that a time series of the integrated IC luminosity has smaller oscillations and slightly longer periods than for the synchrotron radiation.

Future developments of the present work will involve modeling more accurately the particle evolution in the nebula, for example by evolving the distribution function along streamlines. The possibility of a dependence on the polar angle along the TS of the injected particle-distribution function can therefore be investigated. To overcome the problem related to the magneticfield structure and strength, a wider investigation of the parameter space is required, since our results pertain to a single simulation run. If the morphology is found again, a problem intrinsic to the adopted axisymmetric geometry would be highlighted and 3D simulations should be attempted.

This analysis could also be extended to PWNe other than the Crab Nebula, even in different evolutionary stages (e.g. Vela). We believe that the powerful diagnostic techniques described here, which complete those presented in Del Zanna et al. (2006), are sufficiently versatile to be applied to other classes of nonthermal emitting sources.

Acknowledgements. The authors thank Rino Bandiera, for interesting discussions, and the anonymous referee for helpful suggestions. This work has been partly supported by the Italian Ministry of University and Research under PRIN MIUR 2006 (Pacini), by ASI-INAF 2007 (Bandiera). N. Bucciantini was supported by NASA through Hubble Fellowship grant HST-HF-01193.01-A, awarded by the Space Telescope Science Institute, which is operated by the Association of Universities for Research in Astronomy, Inc., for NASA, under contract NAS 5-26555.

\section{References}

Aharonian, F. A. 2007, Science, 315, 70

Aharonian, F. A., Akhperjanian, A., Beilicke, M., et al. 2004, ApJ, 614, 897 Aharonian, F., Akhperjanian, A. G., Aye, K.-M., et al. 2005a, A\&A, 432, L25 Aharonian, F., Akhperjanian, A. G., Aye, K.-M., et al. 2005b, A\&A, 435, L17 Aharonian, F., Akhperjanian, A. G., Bazer-Bachi, A. R., et al. 2006a, A\&A, 448, L43

Aharonian, F., Akhperjanian, A. G., Bazer-Bachi, A. R., et al. 2006b, A\&A, 456, 245

Aharonian, F., Akhperjanian, A. G., Bazer-Bachi, A. R., et al. 2006c, A\&A, 460, 365

Aharonian, F. A., Akhperjanian, A. G., Bazer-Bachi, A. R., et al. 2006d, A\&A, 457, 899

Aharonian, F., Akhperjanian, A. G., Bazer-Bachi, A. R., et al. 2007, A\&A, 472, 489

Albert, J., Aliu, E., Anderhub, H., et al. 2008, ApJ, 674, 1037

Amato, E., Guetta, D., \& Blasi, P. 2003, A\&A, 402, 827

Atoyan, A. M., \& Aharonian, F. A. 1996, MNRAS, 278, 525

Baars, J. W. M., \& Hartsuijker, A. P. 1972, A\&A, 17, 172

Bandiera, R., Neri, R., \& Cesaroni, R. 2002, A\&A, 386, 1044 
Bednarek, W., \& Bartosik, M. 2003, A\&A, 405, 689

Begelman, M. C. 1999, ApJ, 512, 755

Blumenthal, G. R., \& Gould, R. J. 1970, Rev. Mod. Phys., 42, 237

Bogovalov, S. V., \& Khangoulian, D. V. 2002, MNRAS, 336, 53

Bogovalov, S. V., Chechetkin, V. M., Koldoba, A. V., \& Ustyugova, G. V. 2005, MNRAS, 358, 705

Bucciantini, N. 2006, in COSPAR, Plenary Meeting, 36th COSPAR Scientific Assembly, 36, 191

Bucciantini, N. 2008, in Am. Inst. Phys. Conf. Ser., 983, 186

Bucciantini, N., \& Del Zanna, L. 2006, A\&A, 454, 393

Bucciantini, N., Quataert, E., Arons, J., Metzger, B. D., \& Thompson, T. A. 2008, MNRAS, 383, L25

De Jager, O. C., \& Harding, A. K. 1992, ApJ, 396, 161

De Jager, O. C., Harding, A. K., Michelson, P. F., et al. 1996, ApJ, 457, 253

Del Zanna, L., Amato, E., \& Bucciantini, N. 2004, A\&A, 421, 1063

Del Zanna, L., Volpi, D., Amato, E., \& Bucciantini, N. 2006, A\&A, 453, 621

Douvion, T., Lagage, P. O., Cesarsky, C. J., \& Dwek, E. 2001, A\&A, 373, 281

Enomoto, R., Tsuchiya, K., Adachi, Y., et al. 2006, ApJ, 638, 397

Gaensler, B. M., \& Slane, P. O. 2006, ARA\&A, 44, 17

Gaensler, B. M., Pivovaroff, M. J., \& Garmire, G. P. 2001, ApJ, 556, L107

Gaensler, B. M., Arons, J., \& Kaspi, V. M. 2002, ApJ, 569, 878

Gallant, Y. A. 2007, Ap\&SS, 309, 197

Gould, R. J. 1965, Phys. Rev. Lett., 15, 577

Gould, R. J. 1979, A\&A, 76, 306

Green, D. A., Tuffs, R. J., \& Popescu, C. C. 2004, MNRAS, 355, 1315

HESS Collaboration: A. Djannati-Atai, De Jager, O. C., Terrier, R., Gallant, Y. A., \& Hoppe, S. 2007 [arXiv:0710.2247]

Helfand, D. J., Gotthelf, E. V., \& Halpern, J. P. 2001, ApJ, 556, 380

Hennessy, G. S., O’Connell, R. W., Cheng, K. P., et al. 1992, ApJ, 395, L13

Hester, J. J., Mori, K., Burrows, D., et al. 2002, ApJ, 577, L49

Jones, F. C. 1968, Phys. Rev. D, 167, 1159
Kargaltsev, O., \& Pavlov, G. G. 2008, in American Institute of Phys. Conf. Ser., 983, 171

Kennel, C. F., \& Coroniti, F. V. 1984a, ApJ, 283, 694

Kennel, C. F., \& Coroniti, F. V. 1984b, ApJ, 283, 710

Kirk, J. G., Lyubarsky, Y., \& Petri, J. 2007 [arXiv: astro-ph/0703116]

Komissarov, S. S., \& Lyubarsky, Y. E. 2003, MNRAS, 344, L93

Komissarov, S. S., \& Lyubarsky, Y. E. 2004, MNRAS, 349, 779

Kuiper, L., Hermsen, W., Cusumano, G., et al. 2001, A\&A, 378, 918

Ling, J. C., \& Wheaton, W. A. 2003, ApJ, 598, 334

Lu, F. J., Wang, Q. D., Aschenbach, B., Durouchoux, P., \& Song, L. M. 2002, ApJ, 568, L49

Lyubarsky, Y. E. 2002, MNRAS, 329, L34

Marsden, P. L., Gillet, F. C., Jennings, R. E., et al. 1984, ApJ, 278, L29

Masterson, C., Benbow, W. R., \& Van Eldik. 2005, in Int. Cosmic Ray Conf., 4, 143

Melatos, A., Scheltus, D., Whiting, M. T., et al. 2005, ApJ, 633, 931

Mezger, P. G., Tuffs, R. J., Chini, R., Kreysa, E., \& Gemuend, H.-P. 1986, A\&A, 167,145

Mori, K., Burrows, D. N., Hester, J. J., et al. 2004, ApJ, 609, 186

Much, R., Bennett, K., Buccheri, R., et al. 1995, A\&A, 299, 435

Nolan, P. L., Arzoumanian, Z., Bertsch, D. L., et al. 1993, ApJ, 409, 697

Pavlov, G. G., Teter, M. A., Kargaltsev, O., \& Sanwal, D. 2003, ApJ, 591, 1157

Rybicki, G. B., \& Lightman, A. P. 1979, Radiative processes in astrophysics (New York: Wiley)

Slane, P., Helfand, D. J., van der Swaluw, E., \& Murray, S. S. 2004, ApJ, 616, 403

Spitkovsky, A., \& Arons, J. 2004, ApJ, 603, 669

Strom, R. G., \& Greidanus, H. 1992, Nature, 358, 654

Véron-cetty, M. P., \& Woltjer, L. 1993, A\&A, 270, 370

Weisskopf, M. C., Hester, J. J., Tennant, A. F., et al. 2000, ApJ, 536, L81 